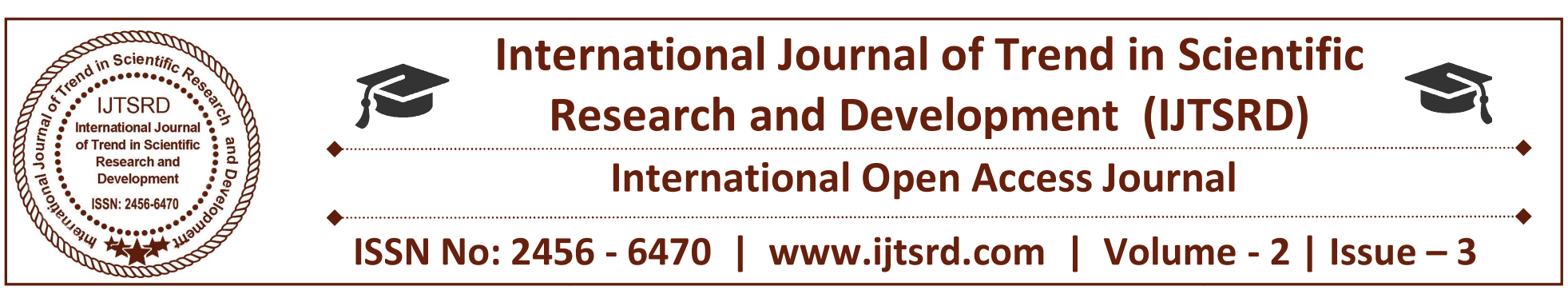

\title{
Brain Tumor Detection Segmentation Techniques
}

\author{
R. Deepa \\ Research Scholar, Department of Computer Science, \\ Gobi Arts \& Science College, Gobichettipalayam, \\ Erode, Tamil Nadu, India
}

\author{
P. Narendran \\ Head of Department, Department of Computer \\ Science, Gobi Arts \& Science College, \\ Gobichettipalayam, Erode, Tamil Nadu, India
}

\section{ABSTRACT}

Computerized brain tumor detection from MRI images is one of the most challenging task in today's contemporary Medical imaging research. Magnetic Resonance Images are used to produce images of soft tissue of human body. It is used to analyze the human organs without the need for surgery. Automatic detection requires brain image most important and challenging aspect of computer aided clinical diagnostic tools. Noises present in the Brain MRI images are multiplicative noises and reductions of these noises are difficult task. The minute anatomical details should not be destroyed by the process of noise removal from clinical point of view. These makes accurate segmentation of brain images a challenge. However, accurate segmentation of the MRI images is very important and crucial for the exact diagnosis by computer aided clinical tools. A large variety of algorithms for segmentation of MRI images had been developed. In this work, it's presented a review of the methods used in brain MRI image segmentation. The review covers imaging modalities, magnetic resonance imaging and methods for noise reduction and segmentation approaches. The paper concludes with a discussion on the upcoming trend of advanced researches in brain image segmentation.

\section{Keywords: Brain, MRI, Segmentation}

\section{INTRODUCTION:}

In the space of image processing, the processing of medical images for medical diagnostics is the prime area of research for many decades and image processing plays key role in the health care. Brain Tumor is hysterical expansion of cancer cells and varied types of brain tumor with different characteristics and treatments. A brain tumor is formed because of abnormal cells created within the brain and brain tumor is primarily classified into two types such as benign tumors and malignant or cancerous tumors. Cancerous tumors further divided into two types' primary tumors that start with in the brain and secondary tumors, brain metastasis which is spread from somewhere else in the body. In the fields of medical, brain tumor grows without any control of typical forces, with the advancement of medical imaging; imaging modalities gain significant part in the brain tumor assessment and huge impact on patient concern. Last few years, promising imaging modalities are CT [Computed Tomography], X-Ray, Single-Photon Emission Computed Tomography (SPECT), Ultrasonography, MRI [Magnetic Resonance Imaging], PET [Positron Emission Tomography],MEG [Magneto Encephalography], and EEG [Electroencephalography].[3]

MR and CT scan images can be used for detection of brain tumor and diagnosis of brain tumor with reliable algorithms is active research area in medical imaging. In medical diagnosis, segmentation of tissues and structures are key component for medical image analysis and Image segmentation plays significant role in diagnosis of brain diseases using the quantitative analysis of MR images such as measuring accurate size and volume of extracted portion of the medical imaging[4]

The brain tumor segmentation taken the research area and two algorithm, there are watershed algorithm, active contour segmentation is relatively compared with other to efficiency one of the approaches is manual while latter one is non-manual. The brain 
tumor segmentation approaches are intended to reveal the exact tumor region and the analysis are carried in different mechanisms, The simulation result show better performance over the traditional states of the methods in an accurate manner. Its diagnosis of proper segmentation method is required for MRI images to carry out and improved diagnosis and treatment. This attempted to review some regression of the worthwhile recent research works done on brain tumor detection, segmentation and area calculation from brain MRI images. A comparative analysis of watershed algorithm and active contour segmentation is performed to know the best brain Theories and Applications. segmentation algorithm for better analysis. [5]

\section{Segmentation Techniques:}

Segmentation is the process which partitions an image into its constituent regions or objects. Image segmentation is the first step in image evaluation. The goal of image segmentation is to represent the images within the form that's significant and easier to research. image segmentation divides an image into more than one parts and is commonly used to discover objects or different relevant records in virtual images. The maximum basic and important approach in images processing is image segmentation and it is necessary in pattern recognition. The simple concept behind segmentation is to segment images into several clusters. The results of segmentation can be such that, it is viable to discover areas of interest and objects within the unique image. Image segmentation is used in type of applicable inclusive of pattern recognition, object identity, function extraction and medical imaging [6].

\section{IMAGES SEGMENTATION TECHNIQUES:}

\section{Thresholding Method}

The most popular and commonly used method for image segmentation is Thresholding methods. In this method, image pixels are divided with the help of intensity level of an image. This method is mainly used to distinguish the foreground objects from background images. In this objects are lighter than its background. Selection of this method depends upon our prior knowledge. The thresholding method is broadly classified into three categories.[7]
I) th/ Global Thresholding,
II) Variable Thresholding,
III) Multiple Thresholding

The various advantages of thresholding method are as follows:

\section{Simple to implement}

- Fast (especially if repeating on similar images).

- Good for some kinds of images (e.g., documents, controlled lighting

- Disadvantages:

- No guarantees of object coherency-may have holes, extraneous pixels, etc

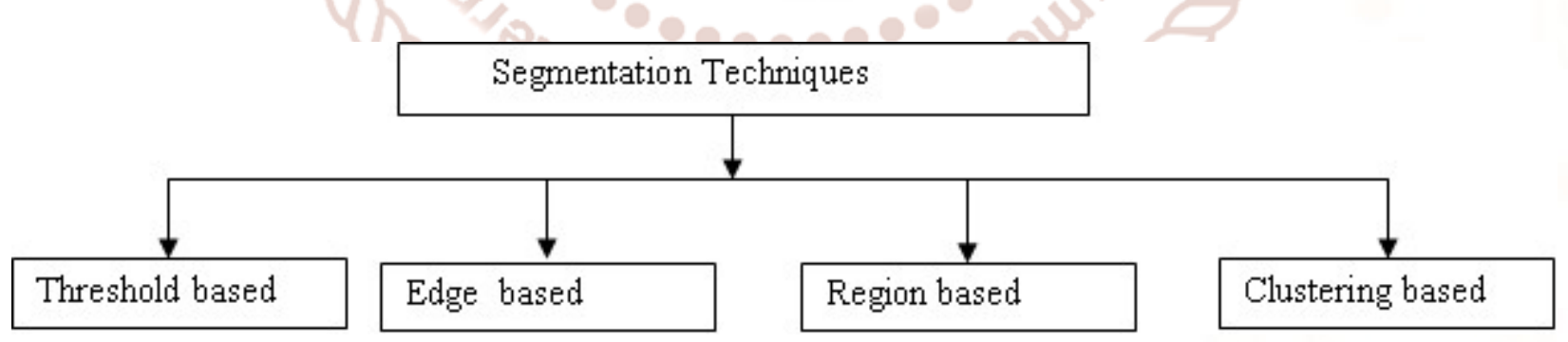

Fig1.1 Segmentation Techniques

\section{Edge based methods}

Edge detection is a full-fledged area in image processing. Region boundaries and edges are somewhat related, the reason is, there is usually a sudden change in intensity at the region boundaries. Edge detection techniques thus can be used as the base for some any other segmentation technique. The edges identified by edge detection are often not properly connected. However, to separate or segment an object from rest of the image there is a requirement of enclosing region boundaries. The desired edges are the boundaries between those objects.[8]

\section{Region based methods}

Region based segmentation is simple as compare to other methods and also noise resilient. It divides an 
image into different regions based on pre-defined criteria, i.e. color, intensity, or object. Region based image segmentation are categorized into three main categories, i.e. region growing, region splitting, and region merging.[9]

\section{Clustering based methods}

The method of clustering organizes the objects into groups based on some feature, attribute and characteristic. Hence a cluster consists of groups of similar objects. There are two types of clustering, supervised and unsupervised. In supervised type clustering, cluster criteria are specified by the user. In unsupervised type, the cluster criteria are decided by the clustering system itself.

\section{1) K-Means Clustering:}

K-Means Clustering partition the $\mathrm{n}$ observations into $\mathrm{k}$ clusters in which each pixel belongs to the clusters by minimizing an objective function in a way that the within cluster sum of squares is get minimized. It starts with initial $\mathrm{K}$ cluster centers and it reassigns the observations to clusters based on the similarity between the observations and cluster/centre. Automation of detection and segmentation of brain tumors in MRI images is a very challenging task due to occurrence of high degree of gray-level similarity in the image. T. U. Paul and S. K. Bandhyopadhyay [10] have presented a fully automated two-step segmentation process of brain MRI images. In the first step, skull stripping is performed by generating a skull mask from the MRI image and in the second step, an advanced K-means algorithm improvised by two-level granularity oriented grid based localization process based on standard local deviation is used to segment the image into gray matter, white matter and tumor region and then length and breadth of the tumor is assessed.

\section{2) Fuzzy C-Means clustering:}

Fuzzy C-means (FCM) clustering is a data clustering method in which each data point belongs to a cluster to a degree specified by a membership value. Fuzzy C-Means divides a collection of vectors into c fuzzy groups and finds a cluster centre in each group such that a cost function of dissimilarity measure is minimized[11].

\section{Watershed Segmentation}

The watershed based method uses the concept of topological interpretation. In this the intensity represents the basins having hole in its minima from where the water spills. When water reaches the border of basin the adjacent basins are merged together. To maintain separation between basins dams are required and are the borders of region of segmentation. These dams are constructed using dilation. The watershed methods consider the gradient of image as topographic surface. The pixels having more gradient are represented as boundaries which are continuous[12].

\section{Partial Differential Equation Based Segmentation Method}

The partial differential equation based methods are the fast methods of segmentation. These are appropriate for time critical applications. There are basic two PDE methods: nonlinear isotropic diffusion filter (used to enhance the edges) and convex nonquadratic variation restoration (used to remove noise). The results of the PDE method is blurred edges and boundaries that can be shifted by using close operators. The fourth order PDE method is used to reduce the noise from image and the second order PDE method is used to better detect the edges and boundaries[13]

It is one of the best methods to group pixels of an image on the basis of their intensities. Pixels falling under similar intensities are grouped together. It is a good segmentation technique for dividing an image to separate a tumor from the image Watershed is a mathematical morphological operating tool. Watershed is normally used for checking output rather than using as an input segmentation technique because it usually suffers from over segmentation and under segmentation.[12] For using watershed segmentation different methods are used. Two basic principle methods are given below: 1) the computed local minima of the image gradient are chosen as a marker. In this method an over segmentation occurs. After choosing marker region merging is done as a second step; 2) Watershed transformation using markers utilizes the specifically defined marker positions. These positions are either defined explicitly by a user or they can be determined automatically by using morphological tools.

\section{METHODOLOGY}

Brain tumor and program code will be written and modeled in MAT Lab image processing tool with the 
help of existing algorithms. The methodology followed is shun in detection methodology

A. Tumor Image Database: The 500 US Tumor images of both normal and abnormal kidney are collected from different hospitals of different patients and are stored in database. One of the image is taken from the database and subjected to tumor detection.

B. Image Pre-Processing: The acquired ultrasound (US) image consists of speckle noise and is of low contrast. Due to this, the image quality is may not be good for analyzing. For surgical operations it is very important to identify the location of kidney stone. To overcome speckle noise, and low contrast, preprocessing of image restoration is required.

\section{Image Restoration}

The very purpose of image restoration is to reduce the degradations that are caused during acquisition of US scanning. In this system for proper orientation, level set function is used. By the use of plan curve motion, curve smoothers, shrinks are eventually disappeared [1].

\section{Smoothing and Sharpening}

To obtained optimal resolution in both spatial and frequency domains, Gabor filter is used which act as band pass filter for the local spatial frequency distribution. The standard deviation of the Gaussian function can be varied to adjust degree of smoothening.

\section{Contrast Enhancement:}

To improve contrast and to obtain uniform intensity histogram equalization is used. This approach can be used on whole image or part of an image. In this system, enhancing the contrast of images is done by transforming the values in an intensity image, such that the histogram of the output image approximately matches a specified histogram. The output signal is of same data type as the input signal.

\section{EXISTING METHODOLOGY}

Clustering is the methodology which objectives to institution items into subsets in this kind of fashion that similar objects are grouped collectively, whilst exceptional items belong to one-of-a-kind companies.
Clustering approach is extensively used in biomedical programs especially for mind tumor detection in abnormal magnetic resonance (MR) images. Inside the segmentation of medical snap shots, the goal is to pick out distinct regions, organs and anatomical structures from statistics received via MRI or other clinical imaging technique. The subsequent information is on current clustering techniques used for tumor clusters.[5]

\section{PROPOSED METHODOLOGY}

There are many image segmentation techniques for detecting tumor from MRI images. Watershed is effective and simple technique for tumor extraction. MRI image is taken as input which includes range of brain, a dark black background and signs. This image is convert into grey scale image for segmentation. Segmentation is used to provide division between regions and categories. Similar types of pixels are presented in grey scale and different pixels are presented with different value. Here images are converted into grey scale and then into binary image. Binary image is required to reduce the complexity of data. It provides actual shape and position of the object and discriminates between foreground and background. Tumor segmentation is done on the basis of affected cells. This process is carried out on the basis of different behavior of pixels in brain image. Different behavior is found according to their shape, brightness and color. Feature extraction is done for brightness, shape and texture.

\section{Watershed Segmentation:}

It is based on gradient and used to group pixels of higher intensity. It is used to separate different in image. It divides an image for extracting tumor. Watershed used flooding process on gray image which performed by morphological operator. Watershed finds ridges lines in images where light pixels are high and dark pixels are low. This is useful to identify or mark foreground objects and background location Threshold segmentation: It is commonly used simplest segmentation method for medical images based on intensity and is based on a threshold value. Initially the image is converted into gray scale and then into binary image. In this technique a threshold value $\mathrm{T}$ is selected from binary image. Histogram is frequently used to select $T$ value from binary image. Basically it is used to extract object from background. It provides fast processing speed, easy to manipulate and lesser space for storage. 
Threshold technique is global and local threshold. In global threshold a single value is selected and multiple values are selected in local threshold. Morphological Operator: used on binary image and gray image for removal of holes in foreground and background. It is also used for noise removal from background. Common Morphological Operations: Shrinking the foreground ("erosion"), Expanding the foreground ("dilation"), Removing holes in the foreground ("closing"), Removing stray foreground pixels in background ("opening"), Finding the outline and skeleton of the foreground There are no individual techniques for detecting tumor. For different body part different segmentation techniques are used. A combination of two techniques always shows a better result than other. We used threshold with morphological operator. Steps which are carried out for detection of tumor are enlisted below.

Step 1: Consider MRI scan image

Step2: Convert image into gray image for segmentation

Step 3: Apply sober operator

Step 4: Filtration is applied for enhancement

Step 5: Watershed is applied to extract different pixels from background

Step 6: Apply threshold technique on segmented image

Step 7: Apply morphological operator imitate for extraction

Steps 8: Finally boundaries are implemented for boundaries of tumor

\section{CONCLUSION}

In this work significant amount of analyses has been performed on brain MRI to detect brain anomalous regions with marker based watershed segmentation algorithm. This work demonstrates the possible enhancement of detection of brain tumor with high accuracy of MR Image. Segmentation of brain image is imperative in surgical planning and treatment in the field of medicine. In this work, we have proposed a computer aided system for brain MR image segmentation for detection of tumor location using K-Means clustering algorithm. The proposed brain tumor detection comprises three steps: image acquisition, pre-processing, and $\mathrm{K}$ Means clustering. Able to segment tumor from different brain MR images from our database.

\section{REFERENCE}

1. P.Narendran and R.Deepa " Survey on Brain Tumor Identification Using Segmentation and MRI Images" International Journal of Innovative Research in Computer and Communication Engineering, Vol. 5, Issue 7, July 2017

2. K.Bhama and A.Jagan,"Analysis of MRI based brain tumor identification using segmentation techniques" International Conference on Communication and Signal Processing, April 6-8, 2016, India 2016 IEEE

3. Gang Li , Improved watershed segmentation with optimal scale based on ordered dither halftone and mutual information, Page(s) 296 - 300, Computer Science and Information Technology (ICCSIT), 2010, 3rd IEEE International Conference, 9-11 July 2011.

4. 4. K.V.N. Sravya Hasanthi T. Madhavi Kumari"A Comparative Study Of Watershed And Active Contour Segmentation Of MRI Images For Brain Tumor Area Calculation "International Journal Of Professional Engineering Studies. Volume VII /Issue 5 / DEC 2016

5. Wen-Liange, De-Hua Chen, Mii-Shen Yang, Suppressed fuzzy-soft learning vector quantization for MR Segmentation", Elsevier Ltd, Vol 52, Issue 1,Pag: 33-43, May2011.

6. Er. Anjna, Er.Rajandeep Kaur "Review of Image Segmentation Technique", International Journal of Advanced Research in Computer Science, Volume 8, No. 4, May 2017 (Special Issue)

7. Anjali, Rajkumar Yadav, Kamaldeep Joshi "A Comparative Study of Image Segmentation Methods" International Journal of All Research Education and Scientific Methods (IJARESM) ISSN: 2455-6211, Volume 4, Issue 7, July- 2016,

8. Ms. Kabade Mankarnika Manohar1, Prof. Mrs. A. S. Patil2," A Review on Techniques of Image Segmentation", International Journal of Advanced Research in Computer and Communication Engineering Vol. 5, Issue 3, March 2016

9. T.U Paul and S.K. Bandyopadhyay, "Segmentation of Brain Tumor from Brain MRI Images Reintroducing $\mathrm{K}$ - Means with advanced Dual Localization Method", International Journal of Engineering Research and Applications, June 2012. 
10. Ruchi D. Deshmukh Prof. Chaya Jadhav,” Study of Different Brain Tumor MRI Image

11. Segmentation Techniques" International Journal of Computer Science Engineering and Technology( IJCSET) | April 2014 | Vol 4, Issue 4,133-136.

12. W. X. Kang, Q. Q. Yang and R. R. Liang, "The Comparative Research on Image Segmentation Algorithms", IEEE Conference on ETCS, pp. 703707, 2009.

13. M. Yambal and H. Gupta, "Image Segmentation using Fuzzy C Means Clustering: A survey", International Journal of Advanced Research in Computer and Communication Engineering, Vol. 2.

14. Anam Mustaqeem, Ali Javed, Tehseen Fatima," An Efficient Brain Tumor Detection Algorithm Using Watershed \& Thresholding Based Segmentation" I.J. Image, Graphics and Signal Processing, 2012, 10, 34- 39 Published Online September 2012 in MEC

15. R.Rajeswari ，G.Gunasekaran "tumor detection and segmentation using watershed and hierarchical clustering algorithm "International Journal of Innovative Research in Computer and Communication Engineering.vol.2, Special Issue 5, October 2014

16. Digital Image Processing, by Rafael C. Gonzalez, Richard Eugene Woods,2008,3rd Edition

17. Digital Image Processing Using Mat lab, by Rafael C.Gonzalez, Richard E. Woods, Steven L. Eddins,2ndEdition 2010

18. Fundamentals of Digital Image Processing, by Anil K. Jain, 6thEdition 2001

19. Digital Image Processing, by Bernd Jähne 2ndEdition 2001

20. Graphics and GUIs with MATLAB: Patrick Marchand.(ISBM 0 -8403-9487-2).1999, 2nd edition, crn per Inc.

21. Digital image processing medical application by Geoff Dougherty 1st Editon 2010

22. R.C. Gonzalez, R.E. Woods and S.L.Eddins, "Digital image processing using MATLAB", Second edition, Gates mark publishing, USA, 2009. 